\section{D) Check for updates}

Cite this: Dalton Trans., 2019, 48, 9037

Received 24th April 2019, Accepted 24th May 2019

DOI: 10.1039/c9dt01710k

rsc.li/dalton

\title{
The surface chemistry of metal-organic frameworks and their applications
}

\begin{abstract}
Ross S. Forgan (iD)
Modifying the outer surfaces of metal-organic frameworks (MOFs) has received considerably less attention than functionalization of the bulk, despite the range of physical and chemical properties that can be tuned by controlling MOF surface chemistry. In this Frontier article, we summarise developments over the last five years in both functionalizing and visualizing the outer surfaces of MOFs, with particular focus on their application as surface-modified nanoparticles for drug delivery and in the enhanced self-assembly of hybrid materials.
\end{abstract}

\section{Introduction}

Metal-organic frameworks (MOFs), network materials consisting of organic ligands connected by metal ions or clusters into multi-dimensional frameworks containing potential porosity, ${ }^{1}$ have become one of the most studied classes of materials of the last twenty years. ${ }^{2}$ Applications abound, including gas storage and separations, ${ }^{3-5}$ catalysis, ${ }^{6-9}$ sensing, ${ }^{10-13}$ and drug delivery, ${ }^{14-17}$ primarily as MOFs exhibit exceptional chemical versatility. Desired functionality can be directly installed

WestCHEM School of Chemistry, University of Glasgow, Joseph Black Building, University Avenue, Glasgow G12 8QQ, UK. E-mail: ross.forgan@glasgow.ac.uk

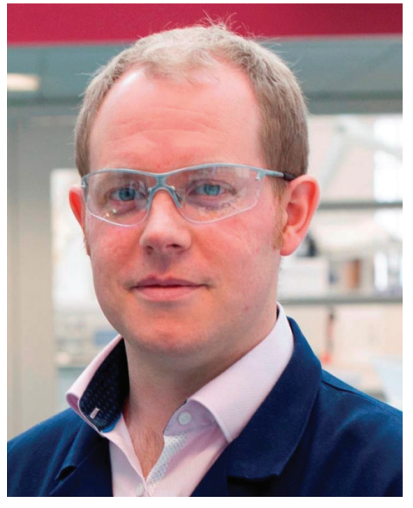

Ross S. Forgan
Dr Ross Forgan is a Royal Society University Research Fellow (2012-) and Reader in Chemistry (2016-) at the University of Glasgow. His research into the application of metal-organic frameworks in biomimetic catalysis and nanoscale drug delivery is underpinned by fundamental studies into molecular recognition and self-assembly processes inside nanoporous materials. In 2016 he won a highly prestigious ERC Starting Grant, and he was awarded the Sessler Early Career Researcher Prize in 2018. He is a Fellow of the Royal Society of Chemistry and a founding member of the Royal Society of Edinburgh Young Academy of Scotland. through the use of bespoke organic linkers or specific metal clusters, ${ }^{18}$ while postsynthetic modification offers a range of possible "upgrades" to basic MOF architectures. ${ }^{19-22}$ Typically, the applications of MOFs rely on chemistry occurring within the pores of the material, and so desired functionality is introduced throughout the bulk of the MOF. In contrast, there is a burgeoning interest in selectively modifying the outer surfaces of MOF particles. $\dagger$ Tuning the chemistry around the pore openings is a potential route to enhance selective guest uptake, and surface functionality can endow enhanced physical properties, such as stability and dispersability, to the MOF material. Approximately five years ago we reviewed the various methodologies for surface modification of MOFs and the impact on a number of applications. ${ }^{23}$ This Frontier article addresses recent advances in the surface chemistry of MOFs in the context of (i) new functionalization protocols, (ii) gaining insights into the processes that occur at MOF surfaces, and (iii) the impact on a range of applications.

\section{New surface modification protocols for biological applications}

Protocols to modify the outer surfaces of MOFs can be broadly divided into two classes: those that occur during self-assembly, and those that occur postsynthetically. ${ }^{23}$ Direct modification during synthesis typically makes use of the coordination modulation protocol - addition of monotopic linkers to solvothermal syntheses - whereby judicious control of synthetic parameters allows the modulators to act as capping agents, ter-

$\dagger$ For many porous materials, the term "surface" is used to describe interior pore surfaces. In this Frontier Article, we use the term to describe the outer particle surface of the $\operatorname{MOF}(\mathrm{s})$ in question. 
minating the coordination polymerization process and being installed primarily on outer surfaces. Postsynthetic methods usually rely on the functionality being incorporated to be larger than the pores of the MOF, thus blocking penetration into the bulk, and attachment can occur via coordination to free metal sites, covalent conjugation at reactive sites, or noncovalent interactions.

\section{Biomolecules}

A number of new innovative routes to surface modified MOFs have been developed of late, driven by the need to functionalise and stabilize MOF nanoparticles for biomedical applications, with particular focus on mild bioconjugate reactions to allow interfacing of MOFs with biomolecules. Lächelt et al. have utilized the coordinative ability of the imidazole sidechain of the amino acid histidine to prepare peptides and proteins terminated by oligohistidine residues - so-called His-tags (Fig. 1a) - which bind strongly to the outer surfaces of MOF

a)

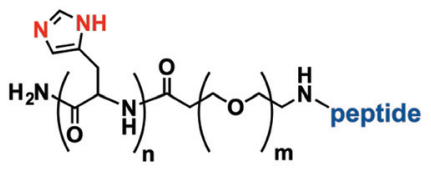

b)

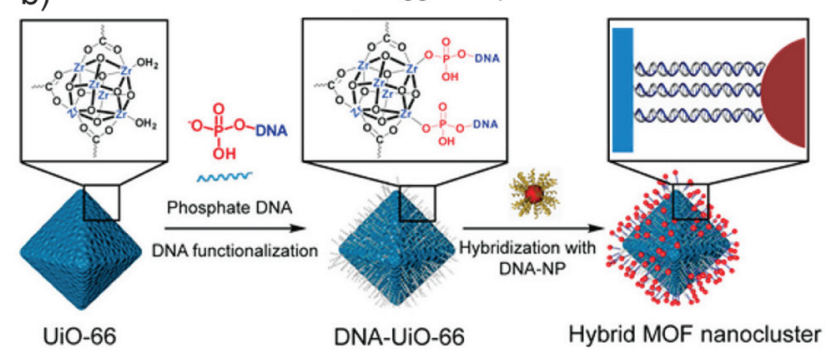

c)

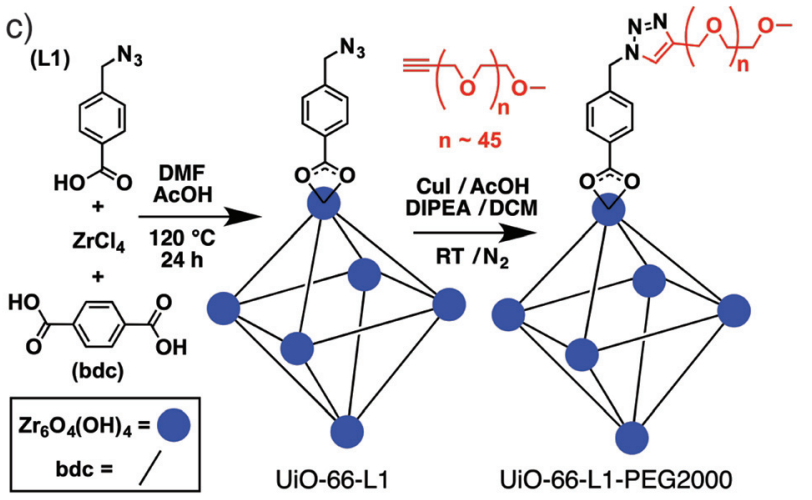

Fig. 1 (a) Chemical structure of a His-tagged peptide that can be coordinated to MOF surfaces for intracellular delivery. (b) Schematic for two stage oligonucleotide functionalisation and hybridization to conjugate inorganic nanoparticles to MOF surfaces. Reproduced with permission from ref. 26 copyright (2017) American Chemical Society. Further permissions related to the material excerpted should be directed to the ACS. (c) Schematic of the "click modulation" protocol whereby UiO-66 is surface-modified by covalent transformations of functionalized modulators. Modified with permission from ref. 31 copyright (2017) Elsevier. nanoparticles. Attachment of dyes to HKUST-1 $\left(\left[\mathrm{Cu}_{3}(\mathrm{BTC})_{2}\right]_{n}\right.$ where $\mathrm{BTC}=1,3,5$-benzenetricarboxylate), MIL-88A(Fe) $\left(\left[\mathrm{Fe}_{3} \mathrm{O}\right.\right.$ (fum $\left.)_{6}\left(\mathrm{H}_{2} \mathrm{O}\right)_{2}(\mathrm{OH})\right]_{n}$, where fum = fumarate) and MOF-801 $\left(\left[\mathrm{Zr}_{6} \mathrm{O}_{4}(\mathrm{OH})_{4}(\mathrm{fum})_{6}\right]_{n}\right)$ shows that binding strength depends on the number of histidine residues in the His-tag; six residues provide stability at $\mathrm{pH}=7.4$, as well as allowing $\mathrm{pH}$ controlled release from the surface. The mild surface attachment protocol - simply immersing the MOF nanoparticles in HEPES-buffered glucose $(\mathrm{pH}=7.4)$ containing the His-tagged molecule means it is highly compatible with biomolecules, allowing the intracellular delivery of pro-apoptotic peptides and proteins attached to MOF-801 with much enhanced cytotoxicity towards HeLa cancer cells. ${ }^{24}$

Specific peptide sequences with high binding affinities for a series of MOF surfaces have been identified by phage display. The MOFs ZIF-8 ([Zn(MeIM) $]_{n}$, where MeIM = 2-methylimidazolate), Fe-BTC (a semi-amorphous solid), and MIL-53(Al)-FA ([AlOH(fum) $]_{n}$ ) were incubated with libraries of 12-mer peptides, and strongly binding candidates identified by multiple cycles of (i) centrifugation of the solids, (ii) washing of the strongly surface-bound peptides away from the MOFs, and (iii) amplification of these peptides by transduction in $E$. coli cultures, followed by sequencing. Identified peptides displayed selective binding for their specific MOFs with dissociation constants as low as $\sim 20 \mu \mathrm{M}$ and enhanced the stability of the MOFs towards hydrolysis. ${ }^{25}$

Attachment of oligonucleotides to MOF surfaces can be achieved by coordinative ${ }^{26}$ or covalent ${ }^{27,28}$ conjugation, with Mirkin et al. developing a general protocol involving phosphate-terminated sequences, which allows direct coordination to a range of MOFs. ${ }^{26}$ The large pore $\mathrm{Zr}$ MOFs NU-1000 $\left(\left[\mathrm{Zr}_{6} \mathrm{O}_{4}(\mathrm{OH})_{8}\left(\mathrm{H}_{2} \mathrm{O}\right)_{4}(\mathrm{TBAPy})_{2}\right]_{n}\right.$, where TBAPy $=4,4^{\prime}, 4^{\prime \prime}, 4^{\prime \prime \prime}-($ pyrene1,3,6,8-tetrayl)tetrabenzoate) and MOF-545 $\left(\left[\mathrm{Zr}_{6} \mathrm{O}_{4}(\mathrm{OH})_{8}\left(\mathrm{H}_{2} \mathrm{O}\right)_{4}\right.\right.$ $\left.\left(\mathrm{TCPP}-\mathrm{H}_{2}\right)_{2}\right]_{n}$, where TCPP- $\mathrm{H}_{2}=$ tetrakis(4-carboxyphenyl)porphyrin, also known as PCN-222) were loaded with insulin and subsequently coated with a 30 base pair, guanine-rich DNA sequence by phosphate coordination. Stability, dispersion, and protein delivery into SKOV-3 human ovarian cancer cells were all enhanced by the protective DNA coating. ${ }^{29}$

When coated with single-stranded DNA, MOFs can be surface functionalized by hybridization with the complementary oligonucleotide strand (Fig. 1b). This technique has allowed installation of a variety of inorganic nanoparticles on the outer surface of MOFs, ${ }^{26}$ including a particular example where surface conjugation of lanthanide-based upconverting nanoparticles improved the efficiency of a porphyrin-containing MOF in photodynamic therapy. ${ }^{30}$ Similarly, DNA-functionalised UiO-68 $\left(\left[\mathrm{Zr}_{6} \mathrm{O}_{4}(\mathrm{OH})_{4}(\mathrm{TPDC})_{6}\right]_{n}\right.$, where TPDC $=p$-terphenyl-4,4"-dicarboxylate), was hybridized with complementary strands containing so-called DNAzymes; sequences that display stimuli-responsive behavior towards certain analytes. Selective release of the drug doxorubicin from the DNA-coated MOFs into MDA-MB-231 breast cancer cells, in response to increased concentrations of both $\mathrm{H}^{+}$and $\mathrm{Mg}^{2+}$ /adenosine triphosphate, led to selective anticancer cytotoxicity in vitro. ${ }^{28}$ 


\section{Polymers}

We have introduced the "click modulation" protocol, a twostep process that is compatible with cargo loading, for the surface modification of Zr MOFs. ${ }^{31}$ Modulators functionalized with reactive groups are introduced into solvothermal syntheses and, with appropriate tuning of synthetic conditions, are installed primarily at nanoparticle surfaces; the protocol allows tuning of both particle size and surface chemistry in one pot. These modulators can then undergo further transformation by mild "click" reactions to selectively modify the MOF surface. Using UiO-66 $\left(\left[\mathrm{Zr}_{6} \mathrm{O}_{4}(\mathrm{OH})_{4}(\mathrm{BDC})_{6}\right]_{n}\right.$, where BDC $=1,4$-benzenedicarboxylate) as an example, it is possible to prepare MOFs decorated with either azides or alkynes and functionalise their surfaces with short-chain alkanes, poly (ethylene glycol) (PEG), poly( $N$-isopropylacrylamide), and poly (L-lactide), for use in drug delivery (Fig. 1c). ${ }^{31,32}$ While enhanced stability and dispersion through incorporation of polymers at particle surfaces is well known, we have shown that functionalization of UiO-66 nanoparticles with different polymers or biomolecules can modify the mechanism by which they are endocytosed by cells. Bare UiO-66 nanoparticles are primarily taken up by HeLa cells through clathrinmediated endocytosis, while the PEGylated MOF nanoparticles favour caveolae-mediated endocytosis. ${ }^{31}$ Coating UiO-66 and later MOF-801 with folic acid also favours caveolae-mediated uptake by targeting specific folate receptors in caveolae invaginations; the result is significantly enhanced in vitro cytotoxicity of the delivered cargo, dichloroacetate, presumably as a consequence of enhanced cytosolic release. ${ }^{32,33}$

Polymer coating of MOF nanoparticles has also been achieved using the GraftFast methodology (Fig. 2a). Aryldiazonium salts are reduced chemically by Fe powder to produce phenyl radicals, which react with the MOF and begin to form a poly(phenylene) sub-layer at the surface. When an acrylate-terminated polymer is included in the process, the acrylate groups are also initiated by the phenyl radicals, leading to polymerization and conjugation with the poly(phenylene) surface and thus the polymer coating of the MOF nanoparticles. Functionalization of MIL-100(Fe) $\left(\left[\mathrm{Fe}_{3} \mathrm{O}(\mathrm{BTC})_{2}\left(\mathrm{H}_{2} \mathrm{O}\right)_{2}(\mathrm{OH})\right]_{n}\right)$ with PEG by this protocol was shown to be highly efficient, with $\sim 30 \% w / w$ polymer incorporation not affecting porosity or drug loading properties. $^{34}$ The radical polymerisation mechanism also makes it broadly applicable to other MOFs. ${ }^{35}$

These examples highlight just some of the recent developments; the enhanced stability of MOFs and use of mild bioconjugate reactions now allows complex multi-step surface functionalization protocols to be achieved..$^{30,36}$

\section{Enhanced assembly of composite materials}

Taking advantage of coordinative and covalent chemistry at the surfaces of MOFs is increasingly being used as a strategy to improve their incorporation into hybrid materials. For example, it has been shown that preparing MOF@MOF composites where the two MOFs are not crystallographically coincident, and so cannot grow epitaxially, can be facilitated by surface-modifying the inner MOF with a coating of poly(vinylpyrrolidone), effectively gluing the two MOFs together through coordinative interactions. ${ }^{37}$ Additionally, polymers containing terephthalate units can exchange with the BDC ligands at the surfaces of MOF-5 $\left(\left[\mathrm{Zn}_{4} \mathrm{O}(\mathrm{BDC})_{3}\right]_{n}\right)$ to produce free-standing monoliths containing up to $80 \% w / w$ of the MOF, enhancing stability and preserving porosity. ${ }^{38}$

Covalently functionalising MOFs with polymers ${ }^{39}$ has been explored as a method for better blending MOF solids into mixed-matrix membranes (MMMs), typically for gas separations. Building on work that has shown that surface modifying UiO-66- $\mathrm{NH}_{2}$ (the isoreticular analogue of UiO-66 with 2-aminoterepthalic acid as linker) with small molecules through amide coupling improves incorporation into polyi-

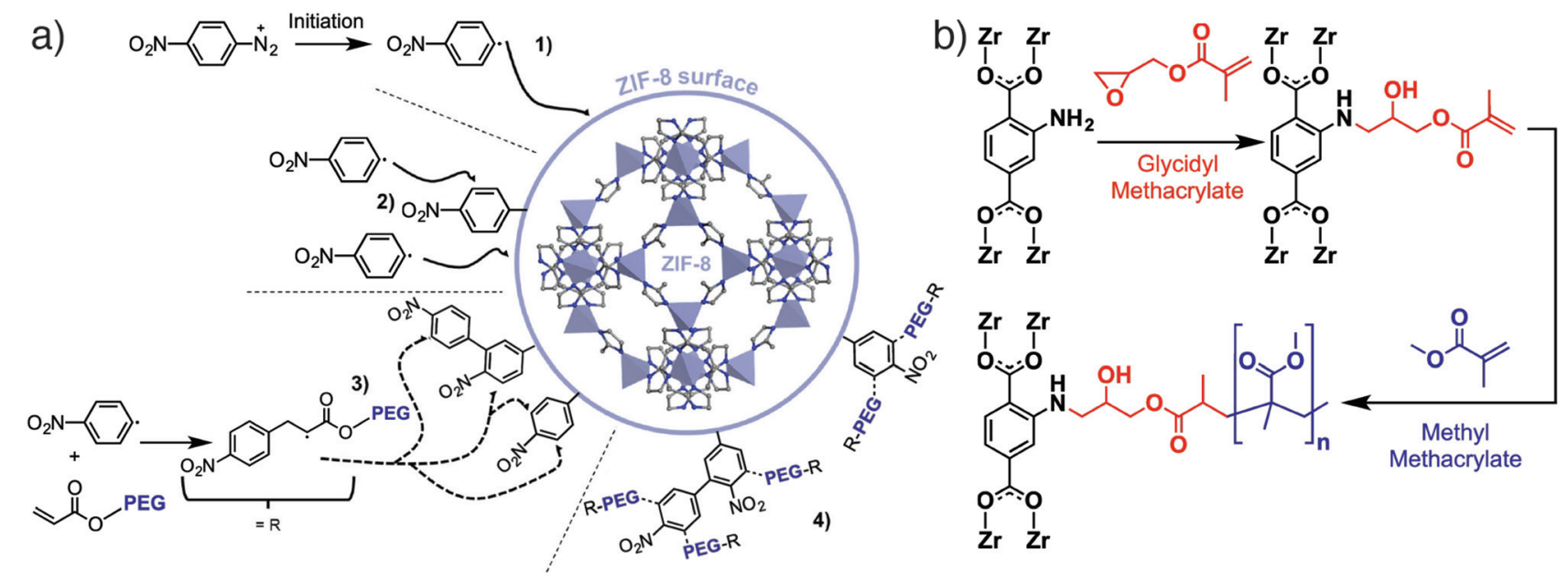

Fig. 2 (a) Schematic of the GraftFast surface polymerization protocol applied to the PEGylation of ZIF-8. Adapted with permission from ref. 35 copyright (2019) American Chemical Society. (b) Surface polymerization directly from UiO-66- $\mathrm{NH}_{2}$ after functionalization with glycidyl methacrylate. 
mides, ${ }^{40}$ a number of surface-based strategies have been employed to blend MOF particles into polymer membranes with improved materials properties and higher MOF loadings. For example, the GraftFast process has been applied to coating ZIF-8 with PEG (Fig. 2a) for improved incorporation into poly (vinyl alcohol) membranes, ${ }^{35}$ while UiO-66 containing pendant allyl groups has been modified with silane-terminated polydimethylsiloxane oligomers that allow enhanced incorporation and crosslinking into a PDMS matrix. ${ }^{41}$

Direct polymerization from MOF surfaces is also being explored. Reacting UiO-66- $\mathrm{NH}_{2}$ with a bulky dianhydride installs anhydrides at the MOF particle surfaces, which can be reacted with diamines and subsequently imidized to generate a polyimide-coated MOF hybrid capable of membrane formation at up to $88 \% w / w$ MOF. Covalent attachment of the MOF to the polymer improved the mechanical properties of the MMM whilst simultaneously enhancing both gas permeability and selectivity for $\mathrm{CO}_{2}$ as the MOF content increases, in contrast to conventional MMMs prepared by physical mixing. ${ }^{42}$

Reacting methacryclic anhydride with amine-tagged MOFs allows installation of methacrylate monomers at the surfaces of the MOFs which can then be polymerized. This has been exploited to prepare MMMs of poly(butylmethacrylate) covalently bound to UiO-66- $-\mathrm{NH}_{2}$ which exhibit enhanced removal of $\mathrm{Cr}(\mathrm{vI})$ from water, ${ }^{43}$ and MMMs of UiO-66- $\mathrm{NH}_{2}$ and methacrylate-crosslinked PEG for enhanced $\mathrm{CO}_{2}$ uptake. ${ }^{44}$ Similarly, conjugation of UiO-66- $\mathrm{NH}_{2}$ with glycidyl methacrylate installs the reactive monomer at the outer surface, allowing polymerisation of poly(methylmethacrylate) (PMMA) directly from the MOF particle (Fig. 2b). Polymer-coated MOF nanoparticles show enhanced stability and dispersion within a PMMA membrane compared to bare UiO-66 and UiO-66- $\mathrm{NH}_{2}$, with improved gas permeability and selectivity in the MMM ascribed to more homogeneous incorporation of the polymercoated MOFs. ${ }^{45}$ Methacrylate-coated MOFs can also be functionalized using atom transfer radical polymerization, ${ }^{46}$ with polymer coatings enhancing MOF stability. ${ }^{47}$ It is clear that, as well as improving stability, enhancing the MOF-polymer interface through control of MOF surface chemistry significantly improves physical and chemical properties of the hybrid material formed.

\section{Direct visualisation of MOF surfaces}

Spectacular advances have been made in the direct visualization of the surface chemistry of MOFs. Han et al. have developed methodology for high-resolution transmission electron microscopy of beam-sensitive samples; typically, MOFs degrade rapidly during imaging, but atomic resolution is now possible (Fig. 3a). UiO-66 nanoparticles show a variety of surface sites (Fig. 3b), with a ligand-terminated (111) surface projecting free carboxylate groups (Fig. 3c), while metalterminated sites are also visible at $(100) /(111)$ kinks between (111) layers, exposing unsaturated $\mathrm{Zr}$ clusters (Fig. $3 \mathrm{~d}$ ). ${ }^{48}$ These
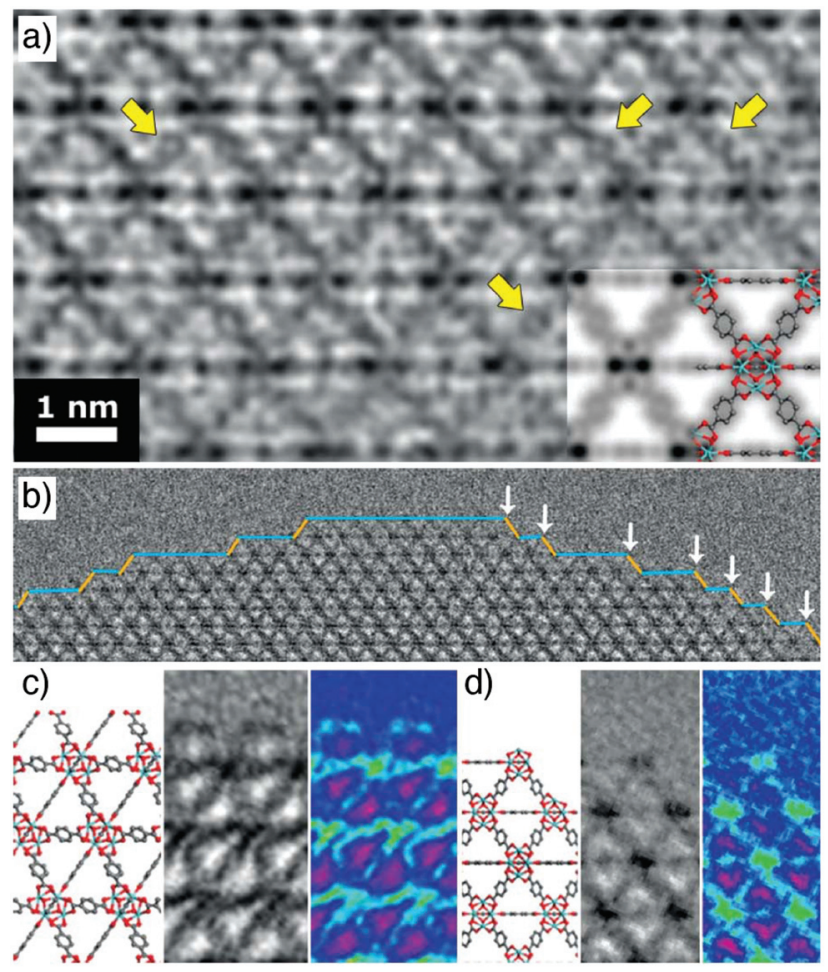

Fig. 3 (a) High resolution TEM image of UiO-66, with yellow arrows indicating atomic resolution of individual benzene rings. (b) Surface termination of a UiO-66 nanoparticle, showing exposed (100) facets (blue line) and (111) facets (yellow line). (100)/(111) kinks are highlighted by white arrows. (c) The carboxylate-terminated (111) surface compared to a structural model. (d) The cluster terminated (100)/(111) kink compared to a structural model. Adapted with permission from ref. 48 copyright (2018) American Association for the Advancement of Science.

functionalities are reactive sites for covalent and coordinative postsynthetic surface modification protocols, respectively.

Dynamic surface processes have also been directly imaged using atomic force microscopy (AFM), in which measurements can be taken at room temperature and under solvent flow. The pillared $\mathrm{MOF} \quad\left[\mathrm{Zn}_{2}(1,4-\mathrm{NDC})_{2}(\mathrm{DABCO})\right]_{n} \quad(1,4-\mathrm{NDC}=1,4-$ naphthalenedicarboxylic acid; DABCO = 1,4-diazabicyclo[2.2.2] octane) can be grown as single crystals with large flat terraces that can be interrogated by AFM at speeds of up to one image every 13 seconds, allowing imaging of guest-responsive dynamics. Imaging the (001) face allows identification of the locations of the paddlewheel clusters with respect to one another and so crystallographic information can be calculated. In the presence of DMF solutions containing the guest molecule biphenyl, uptake of which causes a structural change from tetragonal to orthorhombic symmetry in the bulk crystal, the (001) face could be seen to reversibly deform as the concentration of guest was increased, transforming the lattice parameter from $\gamma=89.6(2)^{\circ}$ to $\gamma=87.3(6)^{\circ}$, compared to the crystallographically determined values of $\gamma=90^{\circ}$ (tetragonal, DMF solvate) and $\gamma=86.1^{\circ}$ (orthorhombic, biphenyl-loaded) for bulk crystals. Addition of a guest with competing donor atoms, e.g., 4,4'-bipyridine or 1,2-di(4-pyridyl)ethylene, caused 
delamination of single layer sheets from the MOF surface, with AFM showing exfoliation occurring by propagation from a surface point defect. ${ }^{49}$

\section{Conclusions and perspectives}

Over the past five years, the understanding of the chemical processes that occur at MOF surfaces has increased significantly, facilitating a range of applications centred on tuning the interaction of MOF particles with their bulk surroundings. Manufacture of hybrid materials and assembly of complex nanoparticulate drug delivery vectors are driving the development of fundamental surface chemistry protocols that are now becoming more generally applicable in nature, yet there still remain significant opportunities in developing, for example, stimuli-responsive systems to control pore openings, with switchable selective cargo uptake and turn-on catalysis just two of the potential applications awaiting the development of new modification protocols. Application of new characterization techniques is also allowing the influence of surface chemistry on other potential applications to begin to emerge; Burdette et al. have used X-ray photoelectron spectroscopy to show that binding of analytes on the surfaces of emissive MOFs is enough to completely quench their fluorescence, casting doubt on the proposed sensing mechanisms, based on bulk analyte intercalation, of a number of MOF materials. ${ }^{50}$ It is clear that modifying the surfaces of MOFs is a powerful protocol for exacting control over bulk physical properties, and a large number of potential applications are beginning to be realized as innovative protocols to control and visualize surface chemistry continue to be developed.

\section{Conflicts of interest}

There are no conflicts to declare.

\section{Acknowledgements}

RSF thanks the Royal Society for a University Research Fellowship, and the European Research Council (ERC) under the European Union's Horizon 2020 Programme for Research and Innovation (grant agreement no. 677289, SCoTMOF, ERC-2015-STG).

\section{Notes and references}

1 H. Furukawa, K. E. Cordova, M. O'Keeffe and O. M. Yaghi, Science, 2013, 341, 1230444.

2 P. Z. Moghadam, A. Li, S. B. Wiggin, A. Tao, A. G. P. Maloney, P. A. Wood, S. C. Ward and D. FairenJimenez, Chem. Mater., 2017, 29, 2618-2625.

3 H. Li, K. Wang, Y. Sun, C. T. Lollar, J. Li and H.-C. Zhou, Mater. Today, 2018, 21, 108-121.
4 B. Li, H.-M. Wen, W. Zhou and B. Chen, J. Phys. Chem. Lett., 2014, 5, 3468-3479.

5 K. Adil, Y. Belmabkhout, R. S. Pillai, A. Cadiau, P. M. Bhatt, A. H. Assen, G. Maurin and M. Eddaoudi, Chem. Soc. Rev., 2017, 46, 3402-3430.

6 S. M. J. Rogge, A. Bavykina, J. Hajek, H. Garcia, A. I. OlivosSuarez, A. Sepúlveda-Escribano, A. Vimont, G. Clet, P. Bazin, F. Kapteijn, M. Daturi, E. V. Ramos-Fernandez, F. X. Llabrés i Xamena, V. Van Speybroeck and J. Gascon, Chem. Soc. Rev., 2017, 46, 3134-3184.

7 A. Dhakshinamoorthy, Z. Li and H. Garcia, Chem. Soc. Rev., 2018, 47, 8134-8172.

8 L. Zhu, X.-Q. Liu, H.-L. Jiang and L.-B. Sun, Chem. Rev., 2017, 117, 8129-8176.

9 A. H. Chughtai, N. Ahmad, H. A. Younus, A. Laypkov and F. Verpoort, Chem. Soc. Rev., 2015, 44, 6804-6849.

10 W. P. Lustig, S. Mukherjee, N. D. Rudd, A. V. Desai, J. Li and S. K. Ghosh, Chem. Soc. Rev., 2017, 46, 3242-3285.

11 Z. Hu, B. J. Deibert and J. Li, Chem. Soc. Rev., 2014, 43, 5815-5840.

12 A. Chidambaram and K. C. Stylianou, Inorg. Chem. Front., 2018, 5, 979-998.

13 L. E. Kreno, K. Leong, O. K. Farha, M. Allendorf, R. P. Van Duyne and J. T. Hupp, Chem. Rev., 2012, 112, 1105-1125.

14 I. Abánades Lázaro and R. S. Forgan, Coord. Chem. Rev., 2019, 380, 230-259.

15 W. Chen and C. Wu, Dalton Trans., 2018, 47, 21142133.

16 P. Horcajada, R. Gref, T. Baati, P. K. Allan, G. Maurin, P. Couvreur, G. Férey, R. E. Morris and C. Serre, Chem. Rev., 2012, 112, 1232-1268.

17 K. Lu, T. Aung, N. Guo, R. Weichselbaum and W. Lin, Adv. Mater., 2018, 30, 1707634.

18 W. Lu, Z. Wei, Z.-Y. Gu, T.-F. Liu, J. Park, J. Park, J. Tian, M. Zhang, Q. Zhang, T. Gentle Iii, M. Bosch and H.-C. Zhou, Chem. Soc. Rev., 2014, 43, 5561-5593.

19 J. D. Evans, C. J. Sumby and C. J. Doonan, Chem. Soc. Rev., 2014, 43, 5933-5951.

20 T. Islamoglu, S. Goswami, Z. Li, A. J. Howarth, O. K. Farha and J. T. Hupp, Acc. Chem. Res., 2017, 50, 805-813.

21 Z. Yin, S. Wan, J. Yang, M. Kurmoo and M.-H. Zeng, Coord. Chem. Rev., 2019, 378, 500-512.

22 R. J. Marshall and R. S. Forgan, Eur. J. Inorg. Chem., 2016, 2016, 4310-4331.

23 C. V. McGuire and R. S. Forgan, Chem. Commun., 2015, 51, 5199-5217.

24 R. Röder, T. Preiß, P. Hirschle, B. Steinborn, A. Zimpel, M. Höhn, J. O. Rädler, T. Bein, E. Wagner, S. Wuttke and U. Lächelt, J. Am. Chem. Soc., 2017, 139, 2359-2368.

25 G. Fan, C. M. Dundas, C. Zhang, N. A. Lynd and B. K. Keitz, ACS Appl. Mater. Interfaces, 2018, 10, 18601-18609.

26 S. Wang, C. M. McGuirk, M. B. Ross, S. Wang, P. Chen, H. Xing, Y. Liu and C. A. Mirkin, J. Am. Chem. Soc., 2017, 139, 9827-9830.

27 W. Morris, W. E. Briley, E. Auyeung, M. D. Cabezas and C. A. Mirkin, J. Am. Chem. Soc., 2014, 136, 7261-7264. 
28 W.-H. Chen, X. Yu, A. Cecconello, Y. S. Sohn, R. Nechushtai and I. Willner, Chem. Sci., 2017, 8, 5769-5780.

29 S. Wang, Y. Chen, S. Wang, P. Li, C. A. Mirkin and O. K. Farha, J. Am. Chem. Soc., 2019, 141, 2215-2219.

30 L. He, M. Brasino, C. Mao, S. Cho, W. Park, A. P. Goodwin and J. N. Cha, Small, 2017, 13, 1700504.

31 I. Abánades Lázaro, S. Haddad, S. Sacca, C. Orellana-Tavra, D. Fairen-Jimenez and R. S. Forgan, Chem, 2017, 2, 561578.

32 I. Abánades Lázaro, S. Haddad, J. M. Rodrigo-Muñoz, C. Orellana-Tavra, V. del Pozo, D. Fairen-Jimenez and R. S. Forgan, ACS Appl. Mater. Interfaces, 2018, 10, 5255-5268.

33 I. Abánades Lázaro, S. Haddad, J. M. Rodrigo-Muñoz, R. J. Marshall, B. Sastre, V. del Pozo, D. Fairen-Jimenez and R. S. Forgan, ACS Appl. Mater. Interfaces, 2018, 10, 3114631157.

34 M. Giménez-Marqués, E. Bellido, T. Berthelot, T. SimónYarza, T. Hidalgo, R. Simón-Vázquez, Á. GonzálezFernández, J. Avila, M. C. Asensio, R. Gref, P. Couvreur, C. Serre and P. Horcajada, Small, 2018, 14, 1870182.

35 M. Benzaqui, R. Semino, F. Carn, S. R. Tavares, N. Menguy, M. Giménez-Marqués, E. Bellido, P. Horcajada, T. Berthelot, A. I. Kuzminova, M. E. Dmitrenko, A. V. Penkova, D. Roizard, C. Serre, G. Maurin and N. Steunou, ACS Sustainable Chem. Eng., 2019, 7, 6629-6639.

36 T. Rijnaarts, R. Mejia-Ariza, R. J. M. Egberink, W. van Roosmalen and J. Huskens, Chem. - Eur. J., 2015, 21, 10296-10301.

37 Y. Gu, Y.-n. Wu, L. Li, W. Chen, F. Li and S. Kitagawa, Angew. Chem., Int. Ed., 2017, 56, 15658-15662.
38 V. J. Pastore, T. R. Cook and J. Rzayev, Chem. Mater., 2018, 30, 8639-8649.

39 T. Kitao, Y. Zhang, S. Kitagawa, B. Wang and T. Uemura, Chem. Soc. Rev., 2017, 46, 3108-3133.

40 S. R. Venna, M. Lartey, T. Li, A. Spore, S. Kumar, H. B. Nulwala, D. R. Luebke, N. L. Rosi and E. Albenze, J. Mater. Chem. A, 2015, 3, 5014-5022.

41 Y. Katayama, K. C. Bentz and S. M. Cohen, ACS Appl. Mater. Interfaces, 2019, 11, 13029-13037.

42 H. Wang, S. He, X. Qin, C. Li and T. Li, J. Am. Chem. Soc., 2018, 140, 17203-17210.

43 Y. Zhang, X. Feng, H. Li, Y. Chen, J. Zhao, S. Wang, L. Wang and B. Wang, Angew. Chem., Int. Ed., 2015, 54, 4259-4263.

44 X. Jiang, S. Li, S. He, Y. Bai and L. Shao, J. Mater. Chem. A, 2018, 6, 15064-15073.

45 H. Molavi, A. Shojaei and S. A. Mousavi, J. Mater. Chem. A, 2018, 6, 2775-2791.

46 K. A. McDonald, J. I. Feldblyum, K. Koh, A. G. Wong-Foy and A. J. Matzger, Chem. Commun., 2015, 51, 1199411996.

47 S. He, H. Wang, C. Zhang, S. Zhang, Y. Yu, Y. Lee and T. Li, Chem. Sci., 2019, 10, 1816-1822.

48 D. Zhang, Y. Zhu, L. Liu, X. Ying, C.-E. Hsiung, R. Sougrat, K. Li and Y. Han, Science, 2018, 359, 675-679.

49 N. Hosono, A. Terashima, S. Kusaka, R. Matsuda and S. Kitagawa, Nat. Chem., 2019, 11, 109-116.

50 J. Yan, A. D. Carl, A. R. Maag, J. C. MacDonald, P. Müller, R. L. Grimm and S. C. Burdette, Dalton Trans., 2019, 48, 4520-4529. 